temperature contrast is presumably reflected by, either as cause or effect, the tectonic activity within the geosynclinal region.

The possible presence of temperature variations naturally raises questions about the heat flow in the area although, unfortunately, very few measurements have been made. There is, however, a published heat flow value of 2.0-2.2 $\mu \mathrm{cal} \mathrm{cm}^{-2} \mathrm{~s}^{-1}$ at Spring Valley and one

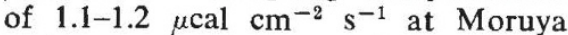
which suggest that towards the middle of the geosyncline at least the heat flow is higher than average in keeping with the enhanced mantle temperature. By the same token, heat flow to the west should be lower, though not necessarily lower than the world average. The only heat flow determinations to the west are several hundred kilometres away at Broken Hill which is itself in a tectonic region, the Adelaide Geosyncline. Tammemagi and Lilley thus predict a heat flow trough between the two geosynclines and, should such a trough be proved, look forward to confirmation of their interpretation of the magnetotelluric data.

\section{CENTRAL NERVOUS SYSTEM \\ Useful Brain Cells}

from our Cell Biology Correspondent Brochemists debating whether or not to take the plunge and tackle some of the biochemical problems posed by the development and differentiation of the central nervous system should be encouraged by what Seeds has to say in the latest issue of the Proceedings of the US National Academy of Sciences (68, 1858; 1971). For he has shown that dissociated mouse embryo brain cells, when put in rotation cultures, form themselves into aggregates which continue to undergo at least some of the biochemical changes that occur during the normal postnatal development of the intact mouse brain.

Seeds chose to follow the activity in his aggregates of three enzymes, choline acetyltransferase, acetylcholinesterase and glutamate decarboxylase, which are associated with the process of nerve transmission and which greatly increase in activity in the mouse brain during the first three to four weeks of postnatal life: the central nervous system of mice is unusually poorly developed at birth and both the formation of synapses and the growth of dendrites as well as biochemical development occur after birth. Seeds finds that the time course and pattern of increase in the activity of choline acetyltransferase and acetylcholinesterase in intact brains and in the aggregates of embryonic brain cells in culture are encouragingly similar.
Moreover, morphological differentiation also occurs in the aggregates; Seeds mentions, for example, the presence of numerous synapses. Although nobody suggests that these aggregates can be used to solve all the questions posed by the development of the central nervous sys'tem they at least offer a most promising starting point.

Brain cells also have attractions for biochemists interested in cytoplasmic microtubules, and Bryan and Wilson (ibid., 1762) report that these organelles in embryonic chick brain cells are polymers of two species of protein not one. Using colchicine as a label, several groups have isolated microtubule subunits from a variety of different cells. The best characterized of these, isolated from adult human brain, appears to be a dimeric molecule comprising two polypeptide chains weighing about 55,000 to 60,000 daltons, and it has generally been assumed that the two polypeptides in each dimer are identical. Bryan and Wilson, however, find that the two polypeptides of the dimer protein of chick brain microtubules can be separated by polyacrylamide gel electrophoresis after they have been reduced and acetylated. Furthermore, although these two polypeptides have the same molecular weight, about 55,000 , they differ significantly in their content of six amino-acids.

From this and similar studies of microtubules from other sources Bryan and Wilson conclude that the basic subunit of these organelles is an $\alpha \beta$ dimer, rather than an $\alpha \alpha$ dimer or a mixture of $\alpha \alpha$ and $\beta \beta$ dimers.

\section{CHEMOSYSTEMATICS \\ New Committee}

A JOINT Committee on Chemotaxonomy has recently been formed by the International Union of Pure and Applied Chemistry (IUPAC) and the International Association for Plant Taxonomy (IAPT) to look into all aspects of the organization of international collaboration in chemosystematics. The committee invites comments from interested persons; these should be sent to the chairman (Dr W. F. Grant, Genetics Laboratory, Macdonald Campus of McGill University, Ste. Anne de Bellevue 800, Quebec, Canada) or to the secretary (Dr T. Swain, Royal Botanic Gardens, Kew, Richmond, Surrey, England).

\title{
Polyadenine and Haemoglobin Messenger
}

LAST year, Lim and Canellakis (Nature, 227, 710; 1970) discovered tracts of polyadenine, some fifty to seventy bases long, in the ribonuclease digests of purified rabbit globin messenger RNA. Since then similar tracts of poly $A$ have been detected in RNA fractions, isolated from the polysomes of HeLa and ascites cells, which are also presumably messengers. It seems therefore that the presence of sequences rich in adenine may be a general feature of many, perhaps even all, eukaryotic messengers. Because of their restricted sequences it has been generally assumed that these tracts are not translated but occur on either, or on one side of the coding sequence in the messenger, and in Nature New Biology next Wednesday Burr and Lingrel report direct evidence that this is the case.

After labelling the $3^{\prime}$ terminal residues of rabbit globin messenger RNAs with tritiated borohydride they digested the molecules with either $T_{1}$ or pancreatic ribonucleases and isolated the $3^{\prime}$ terminal fragments. After $T_{1}$ digestion these fragments elute from 'Sephadex' columns in the region at which oligonucleotides with eight phosphate residues appear. The terminal base of this fragment is adenine. The 3 ' terminal fragments obtained after pancreatic ribonuclease digestion, which cannot contain pyrimidines, are five and six bases long and, because the first guanine residue cannot be within nine bases of this terminus, Burr and Lingrel conclude that these messengers have at their $3^{\prime}$ termini a tract of five or six adenine residues and from the known amino-acid sequences of the rabbit globins it is clear that these bases are not translated.

The sequences Burr and Lingrel have analysed are considerably shorter than those detected by $\mathrm{Lim}$ and Canellakis, and there are several possible explanations of this difference. Much longer poly $A$ sequences may exist at the $5^{\prime}$ ends of these molecules; the short sequence Burr and Lingrel have determined may be part of a much longer adenine rich tract or the poly $A$ tracts may be labile and partially lost either during the extraction of the messenger or indeed in vivo as the molecules age. Needless to say, the function of poly $\mathrm{A}$ in eukaryotic messengers is as yet a matter of speculation and, as anybody who reads what Burr and Lingrel have to say will realize, there is no shortage of ideas. 